INPLASY

PROTOCOL

To cite: Yang et al.

Acupuncture plus tuina for Chronic insomnia: a protocol of a systematic review and meta-analysis. Inplasy protocol 2021100115. doi:

10.37766/inplasy2021.10.0115

Received: 29 October 2021

Published: 29 October 2021

Corresponding author:

Weiwan Yang

ybs18643441852@126.com

Author Affiliation:

Changchun University of

Chinese Medicine

Support:

YDZJ202101ZYTS103.

Review Stage at time of this submission: Preliminary searches.

Conflicts of interest:

None declared.

\section{Acupuncture plus tuina for Chronic insomnia: a protocol of a systematic review and meta-analysis}

Yang, W1; Pan, T2; Guo, X3; Lu, Q4; Wang, H5; Wang, $\mathrm{H}^{6}$.

Review question / Objective: Efficacy and safety of acupuncture combined with tuina in the treatment of patients with chronic insomnia.

Condition being studied: At present, the aging of society is increasingly prominent, the widespread problem of insomnia has aroused the attention of researchers around the world. Insomnia is a common sleep disorder. It refers to a subjective feeling of dissatisfaction with sleep duration and quality that affects social functioning even though there are appropriate opportunities and environments for sleep. Although insomnia is not a serious disease, but often affect people's normal work, life, study and health. Chronic insomnia brings longterm pain to patients, and even forms dependence on sedative sleeping drugs, and can induce or aggravate a variety of physical diseases. With the deepening of the understanding of insomnia in the medical field, insomnia has become the second largest epidemic mental disease in the world as a high risk predictor of mental disorders. Moreover, it will increase the risk of cardiovascular diseases, digestive tract diseases and senile dementia, and even lead to malignant events.

INPLASY registration number: This protocol was registered with the International Platform of Registered Systematic Review and Meta-Analysis Protocols (INPLASY) on 29 October 2021 and was last updated on 29 October 2021 (registration number INPLASY2021100115).

\section{INTRODUCTION}

Review question / Objective: Efficacy and safety of acupuncture combined with tuina in the treatment of patients with chronic insomnia.
Condition being studied: At present, the aging of society is increasingly prominent, the widespread problem of insomnia has aroused the attention of researchers around the world. Insomnia is a common sleep disorder. It refers to a subjective feeling of dissatisfaction with sleep 
duration and quality that affects social functioning even though there are appropriate opportunities and environments for sleep. Although insomnia is not a serious disease, but often affect people's normal work, life, study and health. Chronic insomnia brings long-term pain to patients, and even forms dependence on sedative sleeping drugs, and can induce or aggravate a variety of physical diseases. With the deepening of the understanding of insomnia in the medical field, insomnia has become the second largest epidemic mental disease in the world as a high risk predictor of mental disorders. Moreover, it will increase the risk of cardiovascular diseases, digestive tract diseases and senile dementia, and even lead to malignant events.

\section{METHODS}

Participant or population: Studies on adult patients diagnosed with chronic insomnia are included in this study. No limitations of location, educational background, and gender were imposed.

Intervention: The treatment group was treated with acupuncture plus tuina, acupoints, and frequency were not limited.

Comparator: The control group was oral medication, sham acupuncture, placebo, Chinese herbal medication, physical therapy, and so on, or even with notreatment.

Study designs to be included: RCTs.

Eligibility criteria: Patients suffering from chronic insomnia.

Information sources: Following databases will be searched: Web of Science, PubMed, Cochrane Library, Embase, and Medline databases. In addition, we will also collect four databases of China: China National Knowledge Infrastructure, China Biomedical Literature Database, VIP Database, and Wan-fang Database. We will select the eligible studies published up to October 2021.
Main outcome(s): We use sleep efficiency as the primary outcome of chronic insomnia.

Additional outcome(s): We also care about the following indexes: PSQI, SDRS, ESS, ISI, SDS, PSG, MSLT, MWT as the secondary outcomes.

Quality assessment / Risk of bias analysis: Two reviewers will evaluate the quality of each article using Cochrane Collaboration Tool to assess bias risk. The risk table includes 6 items: random sequence generation mode, whether to use allocation concealment, whether to blind the subjects and intervention providers, whether to blind the results evaluators, whether the results data are complete, whether to select the results report and other bias sources. According to the criteria, it is classified as "low" "unclear" and "high". These even domains will be separately appraised by two reviews, and any differences will be resolved through discussion.

Strategy of data synthesis: ReMan 5.4 software will be used for data synthesis and meta-analysis. All literature will use 12 value of the Chi-Squared test to determine the heterogeneity. When $12<50 \%$, it is considered acceptable. When $12>50 \%$, subgroup analysis should be performed to identify potential causes and record them. When there is homogeneity in the merged outcome results across sufficient studies, a meta-analysis will be conducted. Otherwise, we will carry out a subgroup analysis to explore the causes of obvious heterogeneity.

Subgroup analysis: When meta-analysis shows significant heterogeneity, we will subgroup analysis according to the type of insomnia and different methods of study qualities, interventions, controls, and outcome measurements.

Sensitivity analysis: We conducted a sensitivity analysis according to the recommendations of the Cochrane Handbook. Sensitivity analysis will be used to test the quality of the research contained in the sampled documents. The main 
analysis points included the impact of method quality, sample size, and missing data on the study. The stability of the conclusions can be tested by re-analyzing the conclusions by inputting missing data and changing the type of research.

Country(ies) involved: China.

Keywords: Acupuncture, Tuina, protocol, Chronic insomnia, systematic review.

Contributions of each author:

Author 1 - Weiwan Yang.

Author 2 - Ting Pan.

Author 3 - Xiaole Guo.

Author 4 - Qi LU.

Author 5 - Haili Wang.

Author 6 - Hongfeng Wang. 